\title{
Development of a multistage laser frequency stabilization for an interferometric gravitational-wave detector
}

\author{
Shigeo Nagano ${ }^{\text {a) }}$ \\ Institute for Cosmic Ray Research, University of Tokyo, Kashiwa, Chiba 277-8582, Japan
}

Seiji Kawamura

National Astronomical Observatory of Japan, Mitaka, Tokyo 181-8588, Japan

Masaki Ando

Department of Physics, University of Tokyo, 7-3-1 Hongo, Bunkyo-ku, Tokyo 113-0033, Japan

Ryutaro Takahashi and Koji Arai

National Astronomical Observatory of Japan, Mitaka, Tokyo 181-8588, Japan

Mitsuru Musha

Institute for Laser Science, University of Electro-Communications, Chofugaoka, Chofu, Tokyo 182-8585, Japan

Souichi Telada, Masa-Katsu Fujimoto, Mitsuhiro Fukushima, Yoshihide Kozai, Shoken Miyama, Akitoshi Ueda, Koichi Waseda, and Toshitaka Yamazaki National Astronomical Observatory of Japan, Mitaka, Tokyo 181-8588, Japan

Hideki Ishizuka, Kazuaki Kuroda, Sumihiro Matsumura, Osamu Miyakawa, Shinji Miyoki, Masatake Ohashi, Shuichi Sato, Daisuke Tatsumi, Takayuki Tomaru, and Takashi Uchiyama

Institute for Cosmic Ray Research, University of Tokyo, Kashiwa, Chiba 277-8582, Japan

Keita Kawabe, Naoko Ohishi, Shigemi Otsuka, Atsushi Sekiya, Akiteru Takamori, Shinsuke Taniguchi, Kuniharu Tochikubo, Kimio Tsubono, Kozo Ueda, and Kazuhiro Yamamoto

Department of Physics, University of Tokyo, 7-3-1 Hongo, Bunkyo-ku, Tokyo 113-0033, Japan

Norikatsu Mio and Shigenori Moriwaki

Department of Advanced Materials Science, University of Tokyo, Bunkyo-ku, Tokyo 113-0033, Japan

Gen’ichi Horikoshi, Norihiko Kamikubota, Yujiro Ogawa, Yoshio Saito, and Toshikazu Suzuki

High Energy Accelerator Research Organization, Tsukuba, Ibaragi 305-0801, Japan

Ken'ichi Nakagawa and Ken-ichi Ueda

Institute for Laser Science, University of Electro-Communications, Chofugaoka, Chofu, Tokyo 182-8585, Japan

Akito Araya

Earthquake Research Institute, University of Tokyo, Bunkyo-ku, Tokyo 113-0032, Japan

Nobuyuki Kanda

Department of Physics, Miyagi University of Education, Aoba Aramaki, Sendai 980-0845, Japan

Nobuki Kawashima

Department of Physics, Kinki University, Higashi-Osaka, Osaka 577-8502, Japan

Eiichi Mizuno

Institute of Space and Astronautical Science, Sagamihara, Kanagawa 229-0022, Japan

Mark A. Barton

California Institute of Technology, Mail Stop 18-34, Pasadena, California 91125

Nobuhiro Tsuda

Precision Engineering Division, Tokai University, Hiratsuka, Kanagawa 259-1292, Japan

Namio Matsuda

Tokyo Denki University, Chiyoda-ku, Tokyo 101-8457, Japan

Takashi Nakamura

Yukawa Institute for Theoretical Physics, Kyoto University, Kyoto 606-8502, Japan

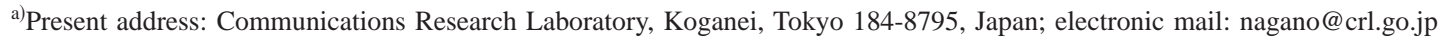


Misao Sasaki, Masaru Shibata, Hideyuki Tagoshi, and Takahiro Tanaka

Department of Earth and Space Science, Osaka University, Toyonaka, Osaka 560-0043, Japan

Ken-ichi Nakao

Graduate School of Science, Kyoto University, Kyoto, 606-8502, Japan

Ken-ichi Oohara

Department of Physics, Niigata University, Niigata, Niigata 950-2102, Japan

Yasufumi Kojima

Department of Physics, Hiroshima University, Higashi-Hiroshima, Hiroshima 739-8526, Japan

Toshifumi Futamase

Astronomical Institute, Tohoku University, Sendai, Miyagi 980-8578, Japan

Hideki Asada

Faculty of Science and Technology, Hirosaki University, Hirosaki, Aomori, 036-8561 Japan

(Received 27 March 2003; accepted 3 June 2003)

Laser frequency stabilization is essential for interferometric gravitational-wave detectors to attain their target sensitivity. We have designed a multistage laser frequency stabilization system which has been applied in the development of the TAMA 300 gravitational-wave detector in Japan. The control topology consisting of two cascaded loops were employed to secure high feedback gain and reliable detector operation and thus allow the best frequency stability and uninterrupted long-term observation. We achieved simultaneously a frequency stability of $5 \times 10^{-5} \mathrm{~Hz} / \sqrt{\mathrm{Hz}}$, and a common-mode rejection ratio (which reduces the coupling of frequency noise to spurious signals in the detector) of $37 \mathrm{~dB}$. The developed system enabled us to operate TAMA 300 with sufficient sensitivity and stability that it had the potential to register gravitational-wave events. The system was confirmed to be suitable for a gravitational-wave detector from the observation run of TAMA 300. (ㄷ) 2003 American Institute of Physics. [DOI: 10.1063/1.1597958]

\section{INTRODUCTION}

Direct detection of gravitational waves (GWs) is confidently expected in the first decade of this century. The detection will open a new window to observe the universe and create the field of GW astronomy. Several projects to build interferometric GW detectors are underway throughout the world. ${ }^{1-4}$ The fundamental configuration of most of these detectors is a Michelson interferometer illuminated by a high-power laser, with three additional elements: FabryPerot arm cavities, a power-recycling system, ${ }^{5}$ and a mode cleaner. ${ }^{6}$ These additional elements increase the detector sensitivity by amplification of the GW signals and reduction of photon shot noise.

The detector sensitivity should be eventually limited by seismic noise, thermal noise, and shot noise. The other noise must be sufficiently suppressed below the goal sensitivity. Among them, laser frequency noise appears in the detector sensitivity coupling with the unavoidable asymmetries of the interferometer such as deviation from resonance and mismatch of the arm cavities. The required frequency stability is typically over $120 \mathrm{~dB}$ higher than the stability of a freerunning laser. A highly stable frequency reference and feedback-control system are necessary to realize the frequency stability required.

A high-finesse optical cavity consisting of a rigid spacer between mirrors makes a stable frequency reference. Many experiments for prestabilization of the laser frequency have been demonstrated with such rigid cavities. ${ }^{7-10}$ Mode cleaners with separately suspended mirrors have also been used as a frequency discriminator in a few studies, ${ }^{11,12}$ which possess higher stability than the rigid cavities in the observation band of GW signals. These demonstrations have shown excellent performance as elements of a system of the frequency stabilization, however, a complete feedback-control topology suitable for actual detectors has not been addressed. The Fabry-Perot arm cavities in the interferometer can also serve as an ultimate frequency reference, since they are highly isolated from seismic motion. Laser frequency stabilization using one arm cavity and appropriate global feedback topology was reported by Kawamura et al. ${ }^{13}$ However, the design and development of a high-performance feedback-control system using the common-motion signal of the arm cavities is necessary for applicability to detectors using the configuration outlined above.

In this article, we present a multistage laser frequency stabilization system for a GW detector. The system was designed considering the practicalities of an interferometric detector and the attainment of the required frequency stability. The system design was actually applied to the development of the laser frequency stabilization system for an interferometric GW detector with $300 \mathrm{~m}$ baseline length (TAMA 300) at the National Astronomical Observatory of Japan in Tokyo. ${ }^{4}$ The system was found to be suitable for advanced interferometric GW detectors such as $\mathrm{LCGT}^{14}$ from the observation run of TAMA 300 .

\section{CONCEPTUAL DESIGN OF A LASER FREQUENCY STABILIZATION SYSTEM FOR A GRAVITATIONAL WAVE DETECTOR}

The conceptual design of the laser frequency stabilization system we proposed for GW detectors is illustrated in 


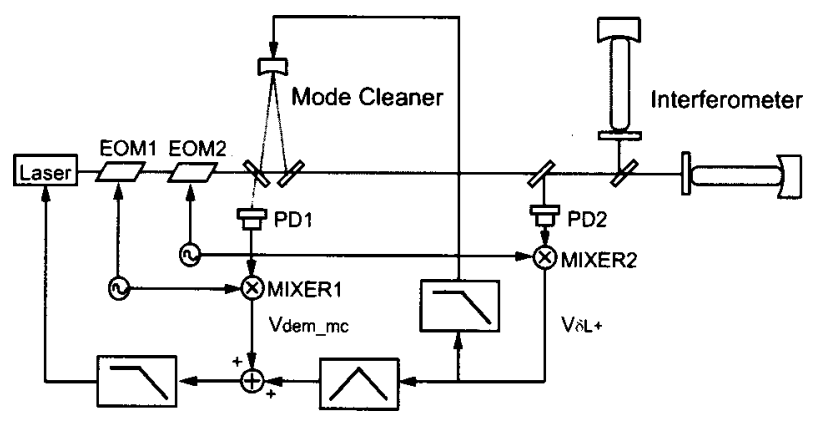

FIG. 1. Conceptual design of a laser frequency stabilization system for a gravitational-wave detector. PD: photodiode, EOM: electro-optical modulator, and $\mathrm{V}$ : voltage signal.

Fig. 1. A multistage frequency stabilization system is employed in the design. It is a control topology consisting of two or more cascaded feedback loops. In this design, the system comprises two cascaded loops: a mode cleaner (MC) servo loop and common-mode cavity length $\left(L_{+}\right)$servo loop. The main role of the MC servo loop is to stabilize the frequency of the light leaving the mode cleaner cavity. The frequency stability of the light transmitted through the mode cleaner is further improved by the $L_{+}$servo loop using the interferometer as a frequency discriminator. Although a Fabry-Perot-Michelson interferometer configuration is assumed here, our design can be easily adapted to a powerrecycled Fabry-Perot-Michelson interferometer as employed in current detectors. ${ }^{1,2,4}$

Such a frequency stabilization system has several advantages for the operation of the detector and the achievement of the frequency-noise level required: simplicity of the system, distribution of the higher attainable feedback gain to two cascaded loops, and elimination of the feedback path to the interferometer.

A simple system is preferable for reliable operation of the GW detector. Using a rigid cavity in the prestabilization loop would give better frequency stability below several $\mathrm{Hz}$ and expectation of easier lock acquisition of the cavity with suspended mirrors, at the price of additional complexity of the control topology. However, long-term stabilization over 1s is not significant for GW detection and the stability of the free-running laser is high enough to acquire lock of the mode cleaner, thus no rigid cavity need be used in our design.

High feedback gain is required to attain the desired frequency stability. However, the control bandwidth cannot be increased enough to allow the necessary gain when the frequency fluctuation is directly stabilized with the resonant frequency of the Fabry-Perot arm cavities in the interferometer, because the control bandwidth is restricted by the freespectral range (FSR) of the cavity. The two cascaded feedback loops can avoid this limitation. Spurious feedback paths can also limit the attainable feedback gain. ${ }^{13}$ The use of electro-optic modulators (EOMs) placed after the mode cleaner causes a spurious feedback path associated with polarization modulation. ${ }^{15}$ It can be circumvented using a feedaround technique which is to sum the error signal of the second stage into that of the first stage. This is often employed in the multistage stabilization systems to expand the control bandwidth without using EOMs for phase correction. ${ }^{13,16}$

The feedback path to the interferometer is eliminated in our design to give adequate sensitivity for GW detection. This strategy avoids the contamination of the differentialmotion signal of the arm cavities $\left(\delta L_{-}\right)$by the error signal of the $L_{+}$servo loop. The coil-magnet actuators attached to the mirror of the arm cavities inevitably apply slightly different forces for the same coil current. The error signal of the $L_{+}$servo loop, which is fed back to the interferometer, couples with the imbalances of the actuator resulting in noise in the $\delta L_{-}$signal.

\section{DESIGN OF MULTISTAGE LASER FREQUENCY STABILIZATION FOR TAMA 300}

The goal sensitivity of TAMA 300 in the first observation phase is $h=1.7 \times 10^{-21} / \sqrt{\mathrm{Hz}}$ in the observation band $(150-450 \mathrm{~Hz})$. A Fabry-Perot-Michelson interferometer is operated without the power recycling technique in this phase. The frequency noise of the light illuminating the interferometer must be suppressed below $5 \times 10^{-5} \mathrm{~Hz} / \sqrt{\mathrm{Hz}}$ to achieve the goal sensitivity, since the common-mode rejection ratio (CMRR) of the interferometer is expected to be $40 \mathrm{~dB}$. The frequency noise is required to be stabilized to 1 $\times 10^{-4} \mathrm{~Hz} / \sqrt{\mathrm{Hz}}$ using the mode cleaner, subsequently the noise of light transmitted through the mode cleaner is further stabilized in the interferometer below the goal sensitivity. In this section, we describe in detail how the above conceptual design was adapted to meet the TAMA 300 requirements.

\section{A. Control topology}

A block diagram of the multistage frequency stabilization system for TAMA300 is shown in Fig. 2. There are two cascaded feedback loops: the MC servo loop and the $L_{+}$ servo loop.

The Pound-Derever-Hall technique is used to extract the control signal of the laser frequency in the MC servo loop. ${ }^{17}$ The technique is represented by a set of blocks consisting of a discriminator (a triangle with "+" and "-" inputs), a cavity pole $\left(C_{\mathrm{mc}}\right)$, and a frequency-voltage converter $\left(D_{\mathrm{mc}}\right)$. The cavity pole is defined as $C_{\mathrm{mc}}=[1$ $\left.+s / \omega_{\mathrm{mc}}\right]^{-1}$, where $s$ is the Laplace frequency variable, and $\omega_{\mathrm{mc}}$ is the half width at half maximum of the cavity resonance in rad/s. The frequency-voltage converter represents the optoelectronic gain of the detection system. Although the error signal of the MC servo loop ( $\left.V_{\text {dem_mc }}\right)$ is shown as fed back to the laser along only one feedback path $\left(F_{\text {feedback }}\right)$, the actual feedback system is composed of three paths to realize a wideband control as explained in Sec. IV.

The frequency of the light transmitted through the mode cleaner $\left(F_{\text {transmitted }}\right)$ is compared with the resonant frequency of the Fabry-Perot arm cavity $\left(F_{\text {int }}\right)$. Here, the PoundDrever-Hall method is modeled in the same manner as in the MC servo, as a set of blocks consisting of a discriminator, an arm cavity pole filter $\left(C_{\text {int }}\right)$, and a frequency-voltage converter $\left(D_{\text {int }}\right)$. The demodulation-voltage signal $\left(V_{\delta L_{+}}\right)$is fed back to the two points: the coil-magnet actuator attached to the end mirror of the mode cleaner, and the error point of 

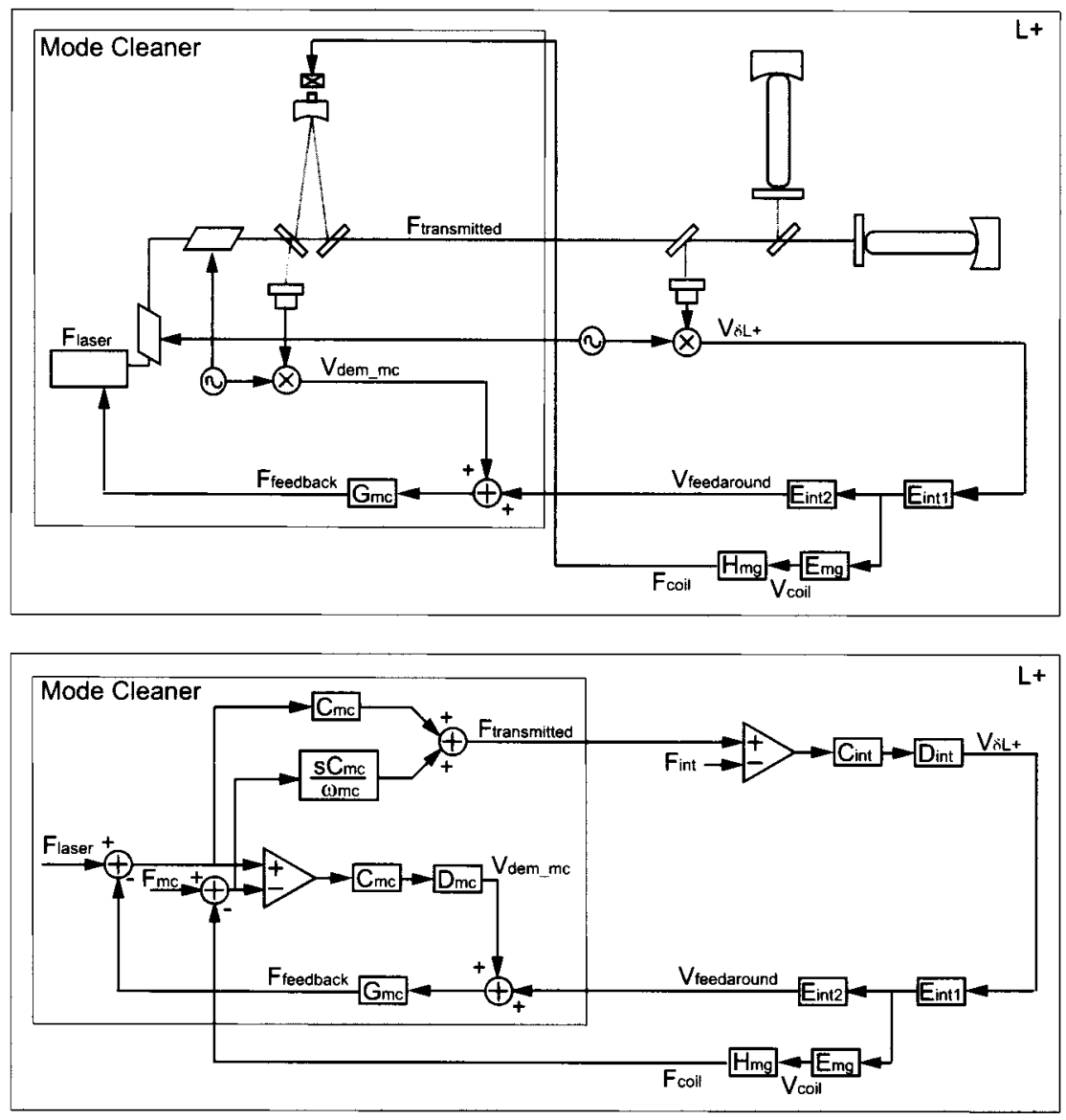

FIG. 2. Block diagram of the laser frequency stabilization system for TAMA300. $C$ : cavity pole, $D$ : frequency-voltage converter, $H$ : voltage-frequency converter, $E$ : filter amplifier, $F$ : frequency, $G$ : $E \times H, V$ : voltage, $s$ : Laplace frequency variable, and $\omega_{\mathrm{mc}}$ : half width at half maximum of the mode cleaner. the MC servo loop. The low frequency components of the $V_{\delta L_{+}}$signal are filter-amplified by electronic servos and directed toward the mode cleaner cavity to stabilize its length fluctuation. This is called the MC feedback path. The high frequency components of the signal are added to $V_{\text {dem_mc }}$ so as to expand the control bandwidth of the $L_{+}$servo loop. This is called the feedaround path. Note that the MC servo loop can be regarded as a "super block" with two input ( $V_{\text {feedaround }}$ and $\left.F_{\text {coil }}\right)$ and one output $\left(F_{\text {transmitted }}\right)$ existing in the $L_{+}$servo loop.

The total transfer function of the $L_{+}$servo loop $\left(T_{L_{+}}\right)$is the composition of two paths:

$$
T_{L_{+}}=T_{\text {MCfeedback }}+T_{\text {feedaround }},
$$

where $T_{\text {MCfeedback }}$ and $T_{\text {feedaround }}$ are the open-loop transfer functions of the MC feedback and feedaround path, respectively. They are given by

$$
\begin{aligned}
T_{\text {MCfeedback }} & =C_{\mathrm{int}} D_{\mathrm{int}} E_{\mathrm{int} 1} E_{\mathrm{mg}} H_{\mathrm{mg}}\left(-\frac{C_{\mathrm{mc}}^{2} D_{\mathrm{mc}} G_{\mathrm{mc}}}{1+T_{\mathrm{mc}}}-\frac{s C_{\mathrm{mc}}}{\omega_{\mathrm{mc}}}\right) \\
& \simeq-C_{\mathrm{int}} D_{\mathrm{int}} E_{\mathrm{int} 1} E_{\mathrm{mg}} H_{\mathrm{mg}}, \\
T_{\text {feedaround }} & =C_{\mathrm{int}} D_{\mathrm{int}} E_{\mathrm{int} 1} E_{\mathrm{int} 2} T_{\text {feedaround(mc) }},
\end{aligned}
$$

where $E$ represents the electronic system for appropriate filtering and amplification, and $H$ is the frequency actuator. The changed resonant frequency of the mode cleaner is reproduced in the frequency of the transmitted light $\left(F_{\text {transmitted }}\right)$ by the function of the servo below the cavity-pole frequency $\left(\omega_{\mathrm{mc}} / 2 \pi\right)$ of the mode cleaner, whereas the change in the mode cleaner resonant frequency directly affects the frequency of the transmitted light above $\omega_{\mathrm{mc}} / 2 \pi{ }^{18,19}$ In Eq. (2), the transfer function from the resonant frequency of the mode cleaner $\left(F_{\text {coil }}\right)$ to $F_{\text {transmitted }}$ is approximated as -1 . This approximation is valid when the open-loop gain of the MC servo loop $\left(T_{\mathrm{mc}}=C_{\mathrm{mc}} D_{\mathrm{mc}} G_{\mathrm{mc}}\right)$ is much larger than unity, as it is below $\omega_{\mathrm{mc}} / 2 \pi$. The transfer function $\left(T_{\text {feedaround(mc) }}\right)$ from the feedaround injection $\left(V_{\text {feedaround }}\right)$ to $F_{\text {transmitted }}$ can be written as

$$
T_{\text {feedaround(mc) }}=\frac{-C_{\mathrm{mc}} G_{\mathrm{mc}}}{1+T_{\mathrm{mc}}} .
$$

This transfer function has a flat frequency response up to around the unity gain frequency of the MC servo loop so that it can be regarded as a wideband frequency actuator. However, it should be noted in respect to the servo design that only a negligible phase delay occurs below roughly one-third of the unity gain frequency.

\section{B. Allowable deviation of carrier frequency for rf intensity noise reduction}

Allowable deviation of the carrier frequency caused by the feedaround technique must be considered to reduce an additional radio-frequency (rf) intensity noise appearing in the light transmitted though the mode cleaner. The intensity noise is converted to a spurious signal by the demodulation process and may preclude the shot-noise-limited operation of the interferometer.

In TAMA 300, the phase modulation sidebands are transmitted through the mode cleaner by making the modulation 

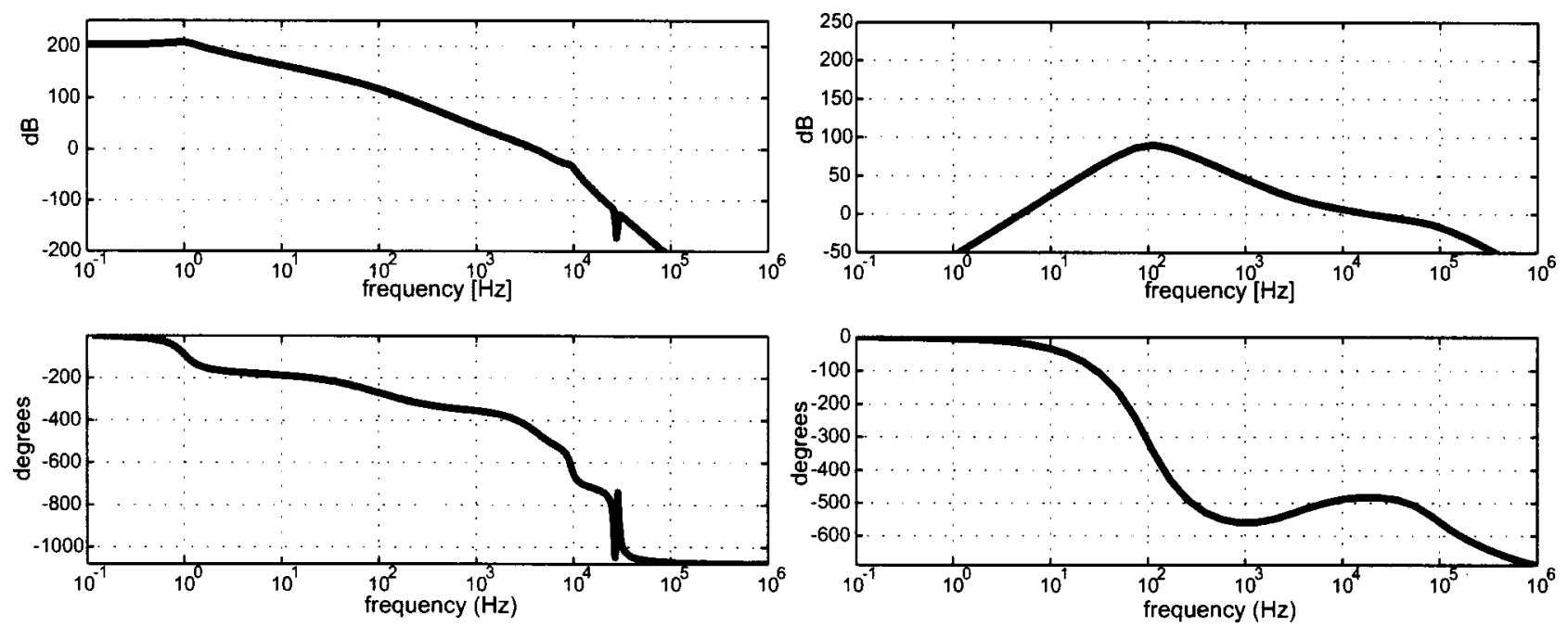

FIG. 3. Bode diagrams of the mode cleaner feedback path $T_{\text {MCfeedback }}$ (left) and the feedaround path $T_{\text {feedaround }}$ (right).

frequency exactly equal to the FSR of the mode cleaner, since an EOM for phase modulation required to control the interferometer is placed before the mode cleaner to avoid wave-front distortion. Additional rf intensity noise is produced within the transmitted light of the mode cleaner, when there exists a deviation of phase modulation frequency from the FSR $(\delta f)$ and that of the carrier frequency from the resonant frequency of the mode cleaner $(\delta \nu)$. This noise mechanism is described in more detail in Refs. 20 and 21. The phase modulation frequency is controlled with the negative feedback to eliminate $\delta f$, whereas $\delta \nu$ can ordinarily be suppressed by the MC servo loop. In a preliminary experiment, ${ }^{22}$ the allowable deviation of the carrier frequency had been measured to be less than $30 \mathrm{~Hz}_{\mathrm{rms}}$ in terms of the root-mean-square (rms) value. However, the feedaround voltage ( $V_{\text {feedaround }}$ ) in the $L_{+}$servo loop shifts the laser frequency away from the resonant frequency of the mode cleaner. This results in a deviation of the carrier frequency ( $\left.\delta \nu_{\text {feedaround }}\right)$ given by

$$
\delta \nu_{\text {feedaround }} \simeq T_{\text {feedaround }} \frac{C_{\mathrm{mc}} \frac{F_{\text {laser }}}{T_{\mathrm{mc}}}+F_{\mathrm{mc}}-F_{\text {int }}}{1+T_{L_{+}}},
$$

where we assume $T_{\mathrm{mc}}$ is much larger than unity. Owing to Eq. (5), $T_{\text {feedaround }}$ serves an important role in decreasing $\delta \nu_{\text {feedaround }}$. The rms value of the $\delta \nu_{\text {feedaround }}$ needs to be less than $30 \mathrm{~Hz}$ in order to suppress the rf intensity noise which is produced by the noise mechanism mentioned above. Therefore this must be taken into account in our servo design.

\section{Servo design}

The MC servo loop had previously been designed and developed for prestabilization of the laser frequency. It was operated with sufficient performance to be utilized in the multistage frequency stabilization system. ${ }^{12}$ The control bandwidth was about $300 \mathrm{kHz}$ and the open-loop gain was more than $120 \mathrm{~dB}$ in the observation band. The frequency stability achieved in the MC servo loop was 1 $\times 10^{-4} \mathrm{~Hz} / \sqrt{\mathrm{Hz}}$.
The crossover frequency between the MC feedback and feedaround path in the $L_{+}$servo loop is set to be $1 \mathrm{kHz}$, which is a trade-off between two considerations. A low crossover frequency results in large values of $V_{\text {feedaround }}$, increasing the rf intensity noise in the transmitted light of the mode cleaner, whereas a higher crossover frequency causes the excitation of the mechanical resonances of the mode cleaner mirror, which could prevent the stable operation of the system. The unity gain frequency of the $L_{+}$servo loop, which is restricted to the feedaround path, is determined to be $20 \mathrm{kHz}$. Although $T_{\text {feedaround(mc) }}$ has a flat frequency dependence up to around the unity gain frequency of the MC servo loop, we chose the unity gain frequency because of the rapid roll-off in phase of $T_{\text {feedaround(mc) }}$.

Figure 3 shows $T_{\text {MCfeedback }}$ and $T_{\text {feedaround }}$. The control system was optimized using a computer simulation. ${ }^{23}$ $T_{\text {MCfeedback }}$ is rolled off quickly above the crossover frequency to remove the influence of the mechanical resonances of the mirror. A notch filter at $27 \mathrm{kHz}$ is also incorporated, since the mechanical $Q$-value of the mirror is estimated to be about $10^{6}$. With respect to the feedaround path, the gain just below the crossover frequency is required to roll off quickly for diminishing the rf intensity noise. $T_{L_{+}}$is plotted in Fig. 4. An open-loop gain of $80 \mathrm{~dB}$ was achieved at $300 \mathrm{~Hz}$, which is large enough to suppress the frequency noise to the required level. The phase margin is approximately $50^{\circ}$ at the unity gain frequency of $20 \mathrm{kHz}$.

Figure 5 shows the estimated deviation of the carrier frequency produced by the injection of $V_{\text {feedaround }}$. The dotted line indicates the expected frequency spectrum of $\delta \nu_{\text {feedaround }}$ calculated by Eq. (5). The rms value of the $\delta \nu_{\text {feedaround }}$ was estimated to be less than $1.1 \mathrm{~Hz}_{\mathrm{rms}}$ (bold line) which satisfies the empirically determined tolerance.

\section{OPERATION OF TAMA 300 WITH MULTISTAGE FREQUENCY STABILIZATION SYSTEM}

This section describes the performance of the system as implemented in TAMA 300. 

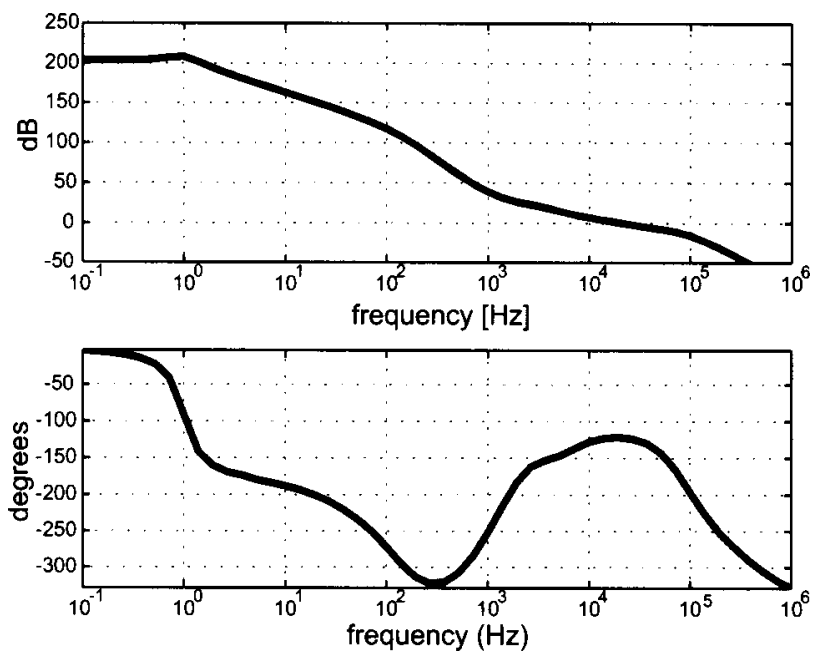

FIG. 4. Open-loop transfer function $\left(T_{L_{+}}\right)$of the $L_{+}$servo loop. This is the composition of the mode cleaner feedback $\left(T_{\text {MCfeedback }}\right)$ and feedaround path ( $\left.T_{\text {feedaround }}\right)$.

\section{A. TAMA 300 gravitational-wave detector}

The schematic diagram of TAMA 300 with the control topology is illustrated in Fig. 6. TAMA 300 comprises a laser, mode cleaner, and a Michelson interferometer with Fabry-Perot arm cavities. Since a light source with high output power and low noise performance is required, we employed an injection-locked Nd:YAG laser with an output power of $10 \mathrm{~W}^{24}$ In order to remove higher modes in the light illuminating the interferometer, an independently suspended triangular ring cavity with a length of $9.75 \mathrm{~m}$ is used as a mode cleaner. ${ }^{20}$ It also serves as a preliminary frequency reference. The reflected light from the mode cleaner is detected by a photodetector to obtain the control signal for the original frequency fluctuation. The signal extracted by the Pound-Derever-Hall technique with phase modulation at 12 MHz is fed back to three actuators: a thermal and PZT tuning

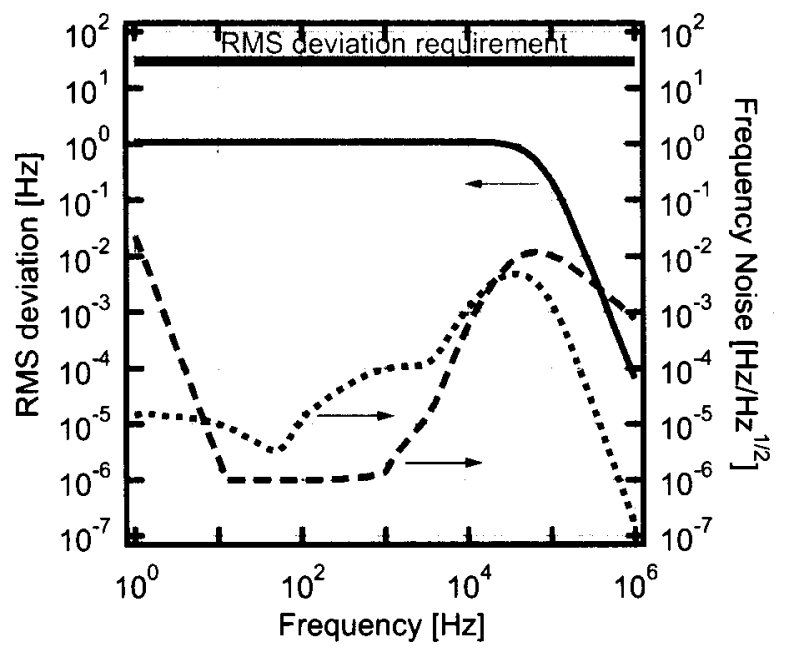

FIG. 5. Calculated rms deviation of the carrier frequency (left axis) and the expected frequency noise (right axis) estimated from the error signal of the $L_{+}$servo. The bold line and dotted line indicate the rms deviation of the carrier frequency and the equivalent frequency spectrum of the feedaround voltage, respectively. The dashed line indicates the expected frequency noise spectrum estimated from the error signal of the $L_{+}$servo. of the master laser and an external EOM. ${ }^{25}$ Although the frequency of the master laser is modulated, the frequency of the injection-locked laser follows that of the master laser within the injection-lock bandwidth. ${ }^{26}$

The phase modulation frequency for controlling the interferometer must be made precisely equal to the FSR of the mode cleaner to allow transmission of the modulation sidebands. The control signal of the modulation frequency is extracted from the light transmitted through an end mirror of the mode cleaner. ${ }^{20}$ The signal is fed back to a voltagecontrolled crystal oscillator (VCXO) for tuning the oscillation frequency to the FSR. This feedback loop compensates for any dc offset or slowly varying deviation of the modulation frequency.

The Fabry-Perot arm cavities have a length of $300 \mathrm{~m}$ and a finesse of 500 to enhance the interaction with GWs. All the interferometer mirrors are isolated from seismic and acoustic noise by three-layer stacks ${ }^{27}$ and double-pendulum suspension systems ${ }^{28}$ inside vacuum chambers. The arm cavities are available as extremely stable references for the laser frequency due to the vibration isolation ratio of over $160 \mathrm{~dB}$. The length control system is designed to operate the interferometer with high sensitivity and stability. The control signals are extracted by the Pound-Drever-Hall technique and the frontal modulation scheme with $15.235 \mathrm{MHz}$ phase modulation. ${ }^{29}$ The $\delta L_{+}$signal is acquired by demodulating the photocurrent from a photodetector placed at the symmetric port of the interferometer.

\section{B. Laser frequency stability}

The measured open-loop transfer function of the $L_{+}$ servo loop is shown in Fig. 7. The control bandwidth of the servo was approximately $20 \mathrm{kHz}$ and the open-loop gain obtained was $80 \mathrm{~dB}$ around the observational band. These are enough to attain the required frequency stability.

Figure 8 shows the error signal spectrum of the $L_{+}$ servo. The high frequency components of the relative frequency noise spectrum estimated from the error signal are low-pass filtered by the mode cleaner cavity so that the frequency noise spectrum is less than the error signal of the MC servo loop even over the control bandwidth of the $L_{+}$servo loop. The frequency noise corresponding to the error signal is $2 \times 10^{-6} \mathrm{~Hz} / \sqrt{\mathrm{Hz}}$ at $300 \mathrm{~Hz}$. However, the real relative frequency stability could be limited by shot noise which may not be reflected in the error signal because of the loop gain suppression. The laser power detected for the $L_{+}$servo loop was about $38 \mathrm{~mW}$ at operation. The shot-noise level was measured to be $5 \times 10^{-5} \mathrm{~Hz} / \sqrt{\mathrm{Hz}}$, dominating the relative frequency stability below $4 \mathrm{kHz}$. The bump around $30 \mathrm{kHz}$ is caused by the small phase margin of the feedback loop.

The laser frequency noise generates spurious signals at the detector output through coupling with the interferometer imperfections. The CMRR of the TAMA 300 interferometer was measured to be $37 \mathrm{~dB}$, which was satisfactorily close to the expected one of $40 \mathrm{~dB}$. Accordingly, the frequency noise stabilized by this developed system posed no significant limitation on achieving the goal sensitivity. 


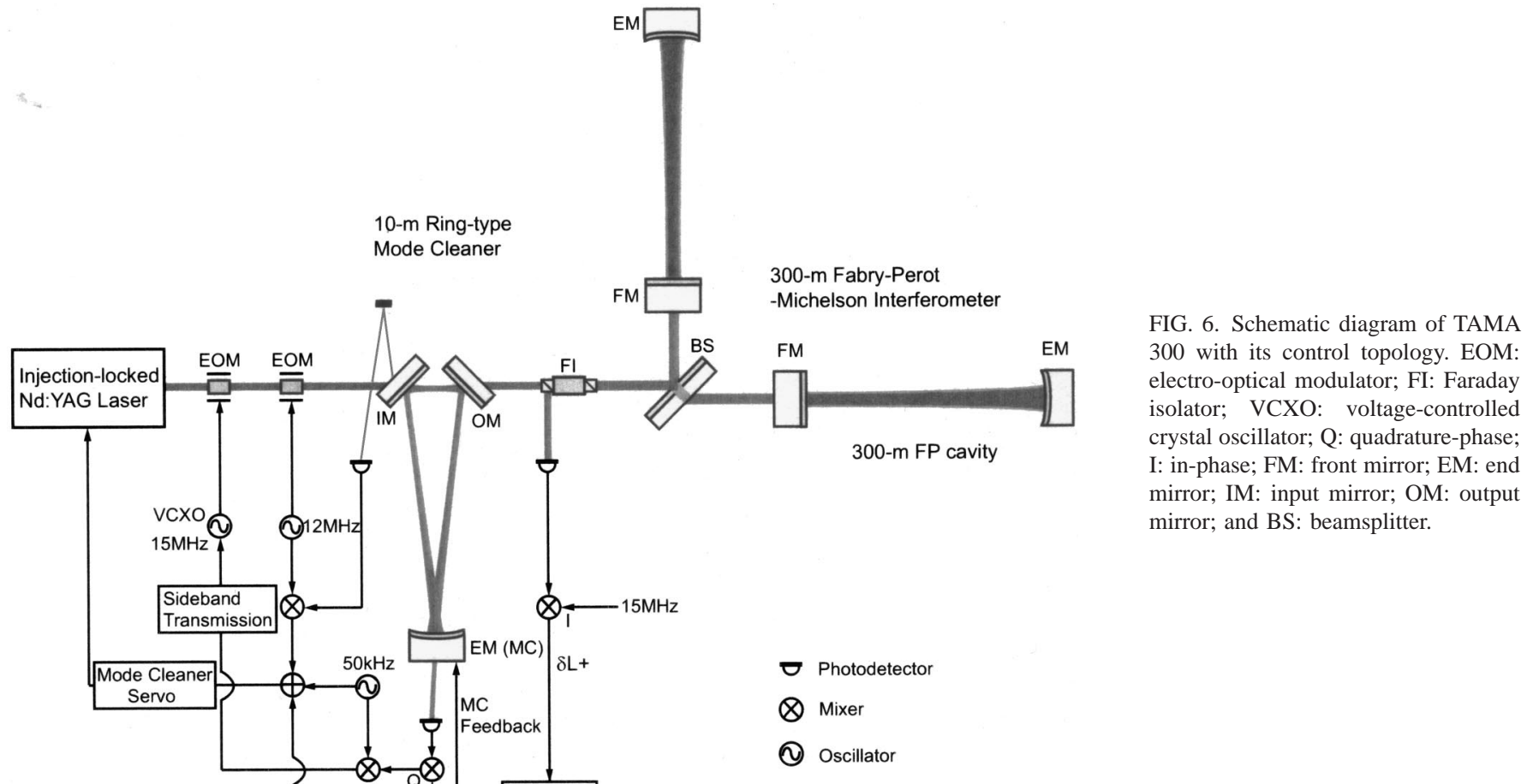

\section{Long-term stability}

The long-term reliability of the system was tested during the observation run of TAMA $300 .{ }^{4}$ The drift length between the mirrors of the Fabry-Perot arm cavity was measured to be less than $50 \mu \mathrm{m}$ by an absolute length determination. ${ }^{30}$ This confirms that the feedback path to the interferometer is not necessary to maintain stable locking of the arm cavity, since the frequency change induced by the drift can be easily compensated by the coil-magnet actuator of the mode cleaner. This avoids the contamination of the $\delta L_{-}$signal with the error signal of the $L_{+}$servo loop.

A minor problem encountered was that when using the interferometer as a frequency discriminator, the state of the $L_{+}$servo loop entirely relates with the operation of the interferometer. Thus when the interferometer fell out of lock, a

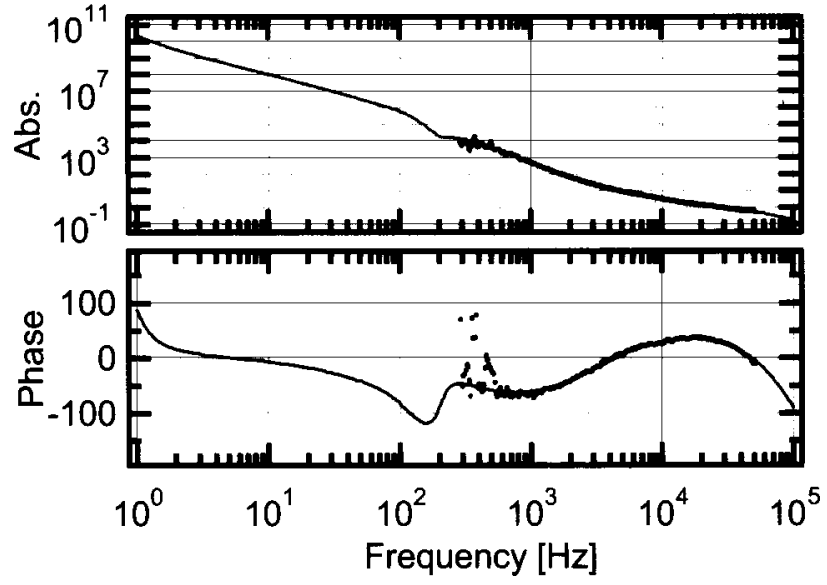

FIG. 7. Measured open-loop transfer function of the $L_{+}$servo loop. The bold and dotted lines are calculated and measured open-loop transfer functions of the $L_{+}$servo loop. large feedback voltage was applied to the error point of the MC servo loop and the coil-magnet actuator of the mode cleaner, which could stop the mode cleaner coming to resonance. To prevent this, voltage limiter circuits were added to the $L_{+}$servo circuits to maintain the stable operation of the mode cleaner.

\section{D. rf intensity noise}

The $L_{+}$servo loop was designed to minimize rf intensity noise in the transmitted light of the mode cleaner. The transmitted light was demodulated at quadrature-phase and inphase to measure the additional $\mathrm{rf}$ intensity noise generated by the feedaround technique. In TAMA 300, the photocurrent

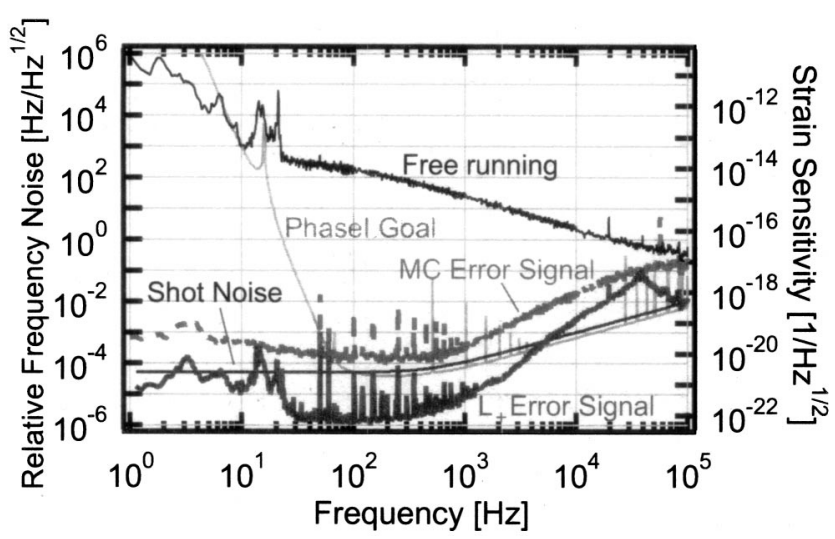

FIG. 8. Relative frequency noise spectrum of the light source (left scale) and corresponding strain sensitivity with the CMRR of $37 \mathrm{~dB}$ (light scale). Thin, dotted, and bold lines indicate the free-running frequency noise, error signal spectrum of the mode cleaner servo loop, and error signal spectrum of the $L_{+}$servo loop. The shot noise indicated is in the $L_{+}$servo loop. The goal sensitivity of TAMA 300 in the first observation phase is shown by a gray line. 
corresponding to the light power at the detector output is expected to be $30 \mathrm{~mA}$ when incident light of $3 \mathrm{~W}$ into the main interferometer and a contrast of $99 \%$ are assumed. The measured rf intensity noise was suppressed below the shotnoise level corresponding to the photocurrent of $30 \mathrm{~mA}$ at the observation band. The spectrum shape of the intensity noise at both demodulation phases had no significant difference from the spectrum reported in Ref. 12, which was observed without the feedaround technique. Thus the system poses no obstacle to achieving shot-noise-limited sensitivity.

\section{ACKNOWLEDGMENT}

The TAMA project is supported by a Grant-in-Aid for Creative Basic Research of the Ministry of Education, Culture, Sports, Science and Technology.

${ }^{1}$ A. Abramovici, W. E. Althouse, R. W. P. Drever, Y. Gürsel, S. Kawamura, F. J. Raab, D. Shoemaker, L. Sievers, R. E. Spero, K. S. Thorne, R. E. Vogt, R. Weiss, S. E. Whitcomb, and M. E. Zucker, Science 256, 325 (1992).

${ }^{2}$ The VIRGO collaboration, VIRGO Final Design Report, 1997.

${ }^{3}$ K. Danzman, H. Lück, A. Rüdiger, R. Schilling, M. Schrempel, W. Winkler, J. Hough, G. P. Newton, N. A. Robertson, H. Ward, A. M. Campbell, J. E. Logan, D. I. Robertson, K. A. Strain, J. R. J. Bennett, V. Kose, M. Kühne, B. F. Schutz, D. Nicholson, J. Shuttleworth, H. Welling, P. Aufmuth, R. Rinkleff, A. Tünnermann, and B. Willke, "Proposal for a 600 $\mathrm{m}$ laser interferometric gravitational wave antenna," Internal Report, MPQ 190, Max-Planck-Institut für Quantenoptik, Garching, Germany, 1994.

${ }^{4}$ M. Ando, K. Arai, R. Takahashi, G. Heinzel, S. Kawamura, D. Tatsumi, N. Kanda, H. Tagoshi, A. Araya, H. Asada, Y. Aso, M. A. Barton, M.-K. Fujimoto, M. Fukushima, T. Futamase, K. Hayama, G. Horikoshi, H. Ishizuka, N. Kamikubota, K. Kawabe, N. Kawashima, Y. Kobayashi, Y. Kojima, K. Kondo, Y. Kozai, K. Kuroda, N. Matsuda, N. Mio, K. Miura, O. Miyakawa, S. Miyama, S. Miyoki, S. Moriwaki, M. Musha, S. Nagano, K. Nakagawa, T. Nakamura, K. Nakao, K. Numata, Y. Ogawa, M. Ohashi, N. Ohishi, S. Okutomi, K. Oohara, S. Otsuka, Y. Saito, M. Sasaki, S. Sato, A. Sekiya, M. Shibata, K. Somiya, T. Suzuki, A. Takamori, T. Tanaka, S. Taniguchi, S. Telada, K. Tochikubo, T. Tomaru, K. Tsubono, N. Tsuda, T. Uchiyama, A. Ueda, K. Ueda, K. Waseda, Y. Watanabe, H. Yakura, K. Yamamoto, and T. Yamazaki, Phys. Rev. Lett. 86, 3950 (2001).

${ }^{5}$ R. W. P. Drever, The Detection of Gravitational Waves, edited by D. G. Blair (Cambridge University Press, Cambridge, England, 1991), p. 306.

${ }^{6}$ A. Rüdiger, R. Schilling, L. Schnupp, W. Winkler, H. Billing, and K. Maischberger, Opt. Acta 28, 641 (1981).

${ }^{7}$ N. M. Sampas, E. K. Gustafson, and R. L. Byer, Opt. Lett. 18, 947 (1993).

${ }^{8}$ N. Uehara and K. Ueda, Opt. Lett. 19, 728 (1994).

${ }^{9}$ K. Nakagawa, A. S. Shelkovnikov, T. Katsuda, and M. Ohtsu, Appl. Opt. 33, 6383 (1994).

${ }^{10}$ F. Bondu, P. Fritschel, C. M. Man, and A. Brillet, Opt. Lett. 21, 582 (1996).

${ }^{11}$ A. Araya, N. Mio, K. Tsubono, K. Suehiro, S. Telada, M. Ohashi, and M.-K. Fujimoto, Appl. Opt. 36, 1446 (1997).
${ }^{12}$ S. Nagano, M. A. Barton, H. Ishizuka, K. Kuroda, S. Matsumura, O. Miyakawa, S. Miyoki, D. Tatsumi, T. Tomaru, T. Uchiyama, M. Ando, K. Arai, K. Kawabe, N. Ohishi, A. Takamori, S. Taniguchi, K. Tochikubo, K. Tsubono, K. Yamamoto, M.-K. Fujimoto, M. Fukushima, S. Kawamura, Y. Kozai, S. Miyama, M. Ohashi, S. Sato, R. Takahashi, S. Telada, T. Yamazaki, N. Mio, S. Moriwaki, G. Horikoshi, N. Kamikubota, Y. Ogawa, Y. Saito, T. Suzuki, M. Musha, K. Nakagawa, A. Ueda, K. Ueda, A. Araya, N. Kanda, T. Nakamura, T. Tanaka, M. Sasaki, H. Tagoshi, T. Futamase, N. Kawashima, E. Mizuno, Y. Kojima, N. Matsuda, K. Oohara, and N. Tsuda, Rev. Sci. Instrum. 73, 2136 (2002).

${ }^{13}$ S. Kawamura, A. Abramovici, and M. E. Zucker, Rev. Sci. Instrum. 68, 223 (1997).

${ }^{14}$ K. Kuroda, M. Ohashi, S. Miyoki, D. Tatsumi, S. Sato, H. Ishizuka, M.-K. Fujimoto, S. Kawamura, R. Takahashi, T. Yamazaki, K. Arai, M. Fukushima, K. Waseda, S. Telada, A. Ueda, T. Shintomi, A. Yamamoto, T. Suzuki, Y. Saito, T. Haruyama, N. Sato, K. Tsubono, K. Kawabe, M. Ando, K.-I. Ueda, H. Yoneda, M. Musha, N. Mio, S. Moriwaki, A. Araya, N. Kanda, and M. E. Tober, Int. J. Mod. Phys. D 8, 557 (1999).

${ }^{15}$ G. C. Bjorklund, Opt. Lett. 5, 15 (1980).

${ }^{16}$ D. Shoemaker, R. Schilling, L. Schnupp, W. Winkler, K. Maischberger, and A. Rüdiger, Phys. Rev. D 38, 423 (1988).

${ }^{17}$ R. W. P. Drever, J. L. Hall, F. V. Kowalski, J. Hough, G. M. Ford, A. J. Munley, and H. Ward, Appl. Phys. B: Photophys. Laser Chem. 31, 97 (1983).

${ }^{18}$ M. J. Lawrence, B. Willke, M. E. Husman, E. K. Gustafson, and R. L. Byer, J. Opt. Soc. Am. B 16, 523 (1999).

${ }^{19}$ J. C. Bienfang, R. F. Teehan, and C. A. Denman, Rev. Sci. Instrum. 72, 3208 (2001).

${ }^{20} \mathrm{~S}$. Telada and the TAMA Collaboration, Proceedings of the XXXIVth Rencontres de Moriond Les Arcs, France, 1999, edited by J. Trân Thanh Vân, J. Dumarchez, S. Reynaud, C. Salomon, S. Thorsett, and J.-Y. Vinet (World Publishers, Hanoi-Vietnam, 2000), p. 213.

${ }^{21}$ R. G. DeVoe and R. G. Brewer, Phys. Rev. A 30, 2827 (1984).

${ }^{22} \mathrm{~S}$. Telada (private communication).

${ }^{23}$ MATLAB for Windows, The Mathworks Inc. Version 5.1, 1997.

${ }^{24}$ S. T. Yang, Y. Imai, M. Oka, N. Eguchi, and S. Kubota, Opt. Lett. 21, 1676 (1996).

${ }^{25}$ G. A. Kerr, N. A. Robertson, J. Hough, and C. N. Man, Appl. Phys. B: Photophys. Laser Chem. B37, 11 (1985).

${ }^{26}$ A. D. Farinas, E. K. Gustafson, and R. L. Byer, J. Opt. Soc. Am. B 12, 328 (1995).

${ }^{27}$ R. Takahashi, F. Kuwahara, E. Mojorana, M. A. Barton, T. Uchiyama, K. Kuroda, A. Araya, K. Arai, A. Takamori, M. Ando, K. Tsubono, M. Fukushima, and Y. Saito, Rev. Sci. Instrum. 73, 2428 (2002).

${ }^{28}$ A. Araya, K. Arai, Y. Naito, A. Takamori, N. Ohishi, K. Yamamoto, M. Ando, K. Tochikubo, K. Kawabe, and K. Tsubono, Proceedings of the TAMA International Workshop on Gravitational Wave Detection, edited by K. Tsubono, M. Fujimoto, and K. Kuroda (Universal Academy, Tokyo, 1997), p. 55.

${ }^{29}$ M. W. Regehr, F. J. Raab, and S. E. Whitcomb, Opt. Lett. 20, 1507 (1995).

${ }^{30}$ A. Araya, S. Telada, K. Tochikubo, S. Taniguchi, R. Takahashi, K. Kawabe, D. Tastumi, T. Yamazaki, S. Kawamura, S. Miyoki, S. Moriwaki, M. Musha, S. Nagano, M.-K. Fujimoto, K. Horikoshi, N. Mio, Y. Naito, A. Takamori, and K. Yamamoto, Appl. Opt. 38, 2848 (1999). 